\title{
Bioökonomie
}

\section{Pilzbiotechnologie als Innovationsmotor}

\author{
VERA MEYER \\ FACHGEBIET ANGEWANDTE UND MOLEKULARE MIKROBIOLOGIE, INSTITUT FÜR BIOTECHNOLOGIE, TU BERLIN
}

DOI: $10.1007 / \mathrm{s} 12268-020-1362-\mathrm{x}$

(C) Springer-Verlag GmbH 2020

Wie stark Pilze und die Pilzbiotechnologie von heute unser tägliches Leben beeinflussen, ist den meisten Menschen nicht bewusst. Pilze sind Meister der Zersetzung: Sie brechen komplexe polymere Substrate sehr effizient in ihre monomeren Bestandteile auf. Sie sind aber auch Meister der Synthese und kombinieren diese Bestandteile zu einem vielfältigen Produktspektrum, das in der Welt der Biologie seinesgleichen sucht. Ohne diese abbauenden und biosynthetischen Fähigkeiten könnten wir viele Lebensmittel und Getränke nicht herstellen, unsere Wäsche nicht bei 30 Grad reinigen, Antibiotika wie Penicillin sowie andere Medikamente wie Immunsuppressiva, Cholesterinsenker oder die Antibabypille nicht verwenden. Und: Wir hätten ohne pilzliche Enzyme keine Biokraftstoffe oder Bioplastik aus nachwachsenden Rohstoffen.

Die Pilzbiotechnologie des 21. Jahrhunderts liefert bereits jetzt viele Lösungsansätze, wie unsere Gesellschaft den Übergang von einer erdölbasierten zu einer nachhaltigen, auf nachwachsenden pflanzlichen Rohstoffen basierten Wirtschaft, der Bioökonomie, schaffen kann [1]. Von der Gesellschaft (noch) nicht beachtet, findet im Stillen - aber unaufhaltsam - ein durch die industrielle Pilzbiotechnologie geprägter Transformationsprozess statt, der die Zukunft unserer Gesellschaft entscheidend mitgestaltet und zur Erreichung der UN-Nachhaltigkeitsziele beitragen wird. Die Pilzbiotechnologie ist daher ein wichtiger Innovationsmotor für die Bioökonomie mit ihren Prinzipien Nachhaltigkeit und Kreislaufwirtschaft [2].

Die Pilzbiotechnologie wird noch weiter an Bedeutung zunehmen und die Bioökonomie maßgeblich stärken. Weltweit erforschen akademische Gruppen und junge Start-ups, wie auf nachhaltigem Wege neuartige pilzbasierte Verbundstoffe für die Textil-, Möbel- oder auch Baustoffindustrie hergestellt werden können, um somit weitere erdölbasierte Produkte $\mathrm{zu}$ ersetzen. Verwendet werden vorrangig Basidiomycota der Gattungen Ganoderma, Pleurotus, Trametes und Fomes, die auf pflanzlichen Abfällen aus der Forst- und Agrarwirtschaft kultiviert werden und im Laufe der Kultivierung einen Verbundwerkstoff (Komposit) ausbilden, der je nach genetischer Ausstattung und Kultivierungsmethode über ähnliche Materialeigenschaften verfügt wie
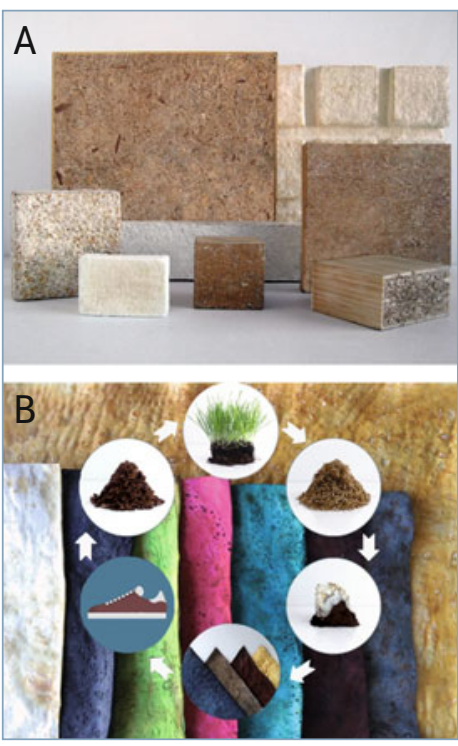

Abb. 1: Pilzbasierte Materialien, entwickelt vom amerikanischen Start-upUnternehmen MycoWorks. A, Pilzkomposite als Baustoffe. B, Pilzleder, als Reishi $^{\mathrm{TM}}$ seit Februar 2020 auf dem Markt. (Abbildung aus [2], Creative Commons Attribution Lizenz, CC BY, http:// creativecommons. org/licenses / by $/ 4.0 /$.

Plastik und elastische Kunststoffe aus der Erdölindustrie [2-4].

Stellen Sie sich vor, in Zukunft könnten wir in Häusern wohnen, die pilzgemacht sind und uns in Räumen bewegen, die mit Pilzmaterialien gedämmt sind, so auch die Innenverkleidung von Autos, Flugzeugen und Raumschiffen. Stühle bestehen aus Pilzmaterialien, unsere Schuhe und Taschen sind aus Pilzleder und damit nicht tierischen Ursprungs (Abb. 1). Dies ist die Vision, die viele von uns Pilzbiotechnologen antreibt! Die Pointe ist hierbei: Wenn diese Dinge nicht mehr benötigt werden, können sie klassisch kompostiert werden. Aus dem landwirtschaftlich genutzten Humus entstehen wieder pflanzliche Reststoffe für pilzbasierte Produkte und Kleidung - der Kreislauf ist damit geschlossen.

Dieser Innovationsprozess in der Pilzbiotechnologie wird meiner Meinung nach zu einer Sprunginnovation führen, die viele Märkte verändern wird. Die Konsequenzen daraus werden weitreichend sein. Neben den Produkten werden sich auch die Berufswelten vieler Menschen ändern, da klassische erdölbasierte Produktionszweige an Bedeutung verlieren oder z. B. tierisches Leder nicht mehr nachgefragt sein wird.

Meines Erachtens ist es notwendig, die Visionen, Möglichkeiten, Chancen, aber auch Risiken der Pilzbiotechnologie der Zukunft schon frühzeitig einer breiten Öffentlichkeit zu vermitteln. Hier ist eine lebendige Wissenschaftskommunikation auf Augenhöhe nach außen in die Gesellschaft, aber auch nach innen, in die Labore hinein gefragt. Hierfür bieten sich Citizen Science-
Formate an, Forschung unter Partizipation von Bürger/innen, die Forschungsthemen mit entwickeln und/oder zur Datenerhebung und Datenauswertung beitragen.

Erste, sehr ermutigende Erfahrungen sammeln wir gerade in unserem transdisziplinären Projekt „Mind the Fungi“, in dem Berliner und Brandenburger Bürger/innen Methoden wissenschaftlichen Arbeitens in der Pilzbiotechnologie nähergebracht und die damit verbundenen Chancen und Herausforderungen offengelegt werden [5]. So finden öffentliche Vortragsreihen, Gesprächsrunden und Workshops zwischen Wissenschaftlern und Künstlern aus den Berlinern Do-It-Yourself-, BioArt- und Citizen Science-Communities statt, und die jeweiligen Sichtweisen auf die Themen Pilze und Pilzbiotechnologie werden transparent und versuchsweise sogar verschränkt. Ein sehr spannender Prozess, mit einer hohen Lernkurve auf allen beteiligten Seiten.

Mehr über dieses Projekt erfahren Sie im Berliner Futurium, dem Haus der Zukünfte, bis Ende 2020 (https://futurium.de/de/feature-art-lab).

\section{Literatur}

[1] Meyer V, Andersen MR, Brakhage AA et al. (2016) Current challenges of research on filamentous fungi in relation to human welfare and a sustainable bio-economy: a white paper. Fungal Biol Biotechnol 3:6

[2] Meyer V, Basenko EY, Benz JP et al. (2020) Growing a circular economy with fungal biotechnology: a white paper. Fungal Biol Biotechnol 7 (im Druck)

[3] Cerimi K, Akkaya KC, Pohl C et al. (2019) Fungi as source for new bio-based materials: a patent review. Fungal Biol for new bio-base

[4] Appels FVW, Camere S, Montalti M et al. (2019) Fabrication factors influencing mechanical, moisture- and water-related properties of mycelium-based composites. Mater Des 161:64-71 [5] Projekt „Mind the Fungi“, TU Berlin, www.mikrobiologie. tu-berlin.de/menue/forschung/mind the fungi/

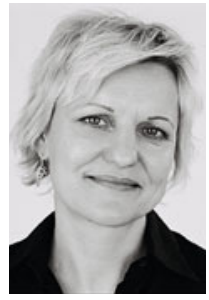

Korrespondenzadresse: Prof. Dr.-Ing. Vera Meyer Fachgebiet Angewandte und Molekulare Mikrobiologie Institut für Biotechnologie Technische Universität Berlin Gustav-Meyer-Allee 25

D-13355 Berlin vera.meyer@tu-berlin.de 